\title{
Soldadura de aleaciones de aluminio con láseres de Nd:YAG de alta potencia
}

\author{
J.A. $\mathrm{G}^{\mathrm{a}} \cdot$-Orza ${ }^{(*)}$ \\ Resumen: El uso del Al y sus aleaciones es creciente en la fabricación de vehículos de transporte. Su soldabi- \\ lidad es menor que la de otros materiales y las aleaciones presentan cierta disminución de propieda- \\ des por pérdida de aleantes y/o de su tratamiento térmico. Se revisa brevemente el uso de láseres de \\ $\mathrm{Nd}$ :YAG de potencia en soldeo de aleaciones de aluminio y se presentan resultados de la combina- \\ ción de haces de potencia guiados por fibras de pequeño diámetro, con focos ligeramente superpues- \\ tos en la pieza. Finalmente, se caracterizan los humos producidos en la soldadura con láser continuo \\ de $2 \mathrm{~kW}$.
}

Palabras clave: Láseres de YAG de alta potencia. Aleaciones de aluminio. Combinación de haces. Seguridad láser. Emisiones.

\section{Aluminium alloys welding with high-power Nd:YAG lasers}

\begin{abstract}
Aluminium alloys have good mechanical properties (high strength-to-weight ratio, corrosion resistance) and good workability. Their applications are growing up, specially in the transportation industry. Weldability is however poorer than in other materials; recent advances in high power YAG lasers are the key to obtain good appearance welds and higher penetration, at industrial production rates. Results of the combination of high power YAG beams with small fiber diameters and specific filler wires are presented. It is also characterized the airborne particulated material, by-product of the laser process: emission rates, size distribution and chemical composition are given for several aluminium alloys.
\end{abstract}

Keywords: High-power YAG laser. Aluminium alloys. Beam combination. Laser safety. Byproducts.

\section{INTRODUCCIÓN}

El aluminio y sus aleaciones encuentran cada vez mayor aplicación en la industria, principalmente en los sectores de automoción, ferroviario y aeroespacial, debido a la reducción de peso que suponen sus construcciones. La relación entre las propiedades mecánicas de estos materiales y su peso es buena, y también lo son la resistencia a la corrosión y su facilidad para ser trabajados.

Estos materiales se sueldan con éxito con diferentes técnicas, pero su soldabilidad presenta algunas dificultades, ya que tienen alta reflectividad y alta conductividad térmica, así como baja viscosidad cuando es líquido y alto coeficiente de dilata-

(*) IMADE (Grupo Láser). Ctra. de Valencia, km. 7. 28031Madrid (España). ción. La pérdida de aleantes de bajo punto de ebullición (p. ej. magnesio en la serie 5000) y la pérdida de propiedades en las aleaciones endurecidas térmicamente son habituales en estas soldaduras.

La aparición, en los últimos años, de fuentes láser de Nd:YAG de alta potencia $(2-4 \mathrm{~kW})$ permite abordar la soldadura de estos materiales con ventajas sobre el $\mathrm{CO}_{2}$.

A continuación, se exponen algunas generalidades del soldeo de aleaciones de aluminio con láseres de Nd:YAG y se presentan resultados de la combinación de haces de alta potencia y de emisiones de materia particulada durante la soldadura de estos materiales. Los trabajos descritos se realizaron en las instalaciones del Institute für Strahlwerkzeuge (IFSW, Univ. de Stuttgart) dentro del proyecto europeo BE-7997, en el que se han llegado a soldar espesores de $5 \mathrm{~mm}$ de aluminio de la serie 6000 , 
con velocidades de $1,5 \mathrm{~m} / \mathrm{min}$ y láseres de $2+2 \mathrm{~kW}$ continuos.

\section{SOLDEO DE ALEACIONES DE ALUMINIO CON LÁSER}

\subsection{Generalidades}

El soldeo láser, como proceso físico, ofrece muchas ventajas por la aplicación de forma controlada de altas densidades de potencia sobre zonas muy definidas de una pieza, con baja cesión total de energía. Esto permite obtener soldaduras estrechas y muy precisas, y zonas de afectación térmica reducidas. La desventaja técnica son los requisitos de ajuste en la colocación de las piezas para su procesado.

Respecto a otros métodos convencionales utilizados en la fabricación de vehículos de transporte, el láser es más rápido que la soldadura por puntos (resistencia) y la TIG. En el caso del aluminio, la técnica de electrodos no es aplicable por la elevada conductividad eléctrica de la unión; la soldadura por arco produce elevadas distorsiones en las piezas.

Se obtienen buenos resultados sobre estos materiales con soldadura láser de $\mathrm{CO}_{2}$, cuando se controla la formación del plasma. Los láseres de Nd:YAG, a pesar de su peor calidad de haz, presentan ventajas como la transmisión del haz láser por fibras ópticas flexibles (de especial interés en procesado 3-D) y la mayor absorción de su radiación (1,06 mm, infrarrojo cercano) en la superficie de los metales. También la óptica necesaria es más barata y convencional que la utilizada con la radiación infrarroja, más lejana, del $\mathrm{CO}_{2}$.

En general, la calidad de la soldadura de aleaciones de aluminio necesita mayor control y estabilidad del proceso que los aceros. Porosidad, agrietamiento en caliente y formación de cavidades son los defectos más importantes.

El soldeo de aleaciones de aluminio necesita de la formación de keyhole; la penetración máxima alcanzable está limitada por la potencia de la fuente láser. En los últimos años, la disponibilidad comercial de potencia media de los láseres de Nd:YAG, ha crecido de $400 \mathrm{~W}$ a $3 \mathrm{~kW}$. Ello ha abierto la aplicación industrial de estas fuentes, más flexibles, a la soldadura de aluminio. Por ejemplo, piezas de 2 $\mathrm{mm}$ soldados a $2 \mathrm{~m} / \mathrm{min}$ con fuentes de $2 \mathrm{~kW}$.

Los desarrollos en las fuentes de Nd:YAG se dirigen a mejorar la calidad del haz y al uso de mayores potencias (mejoras en el intercambio de calor en los resonadores). Es, además, fundamental la aplicación de mecanismos de excitación más eficientes/baratos: lámparas con mayor vida y el futu- ro bombeo con láseres de diodo de potencia. El mercado mundial de equipos de Nd:YAG para procesado de materiales, es de $117 \cdot 10^{6} \$$; el crecimiento estimado para 1998, del $19 \%$ (1), va directamente unido a su uso en la industria de vehículos de transporte.

Comienza a haber datos disponibles de la soldabilidad láser de distintas aleaciones de aluminio. Una mayor introducción de esta técnica en líneas de producción pasa, sin embargo, por los altos costes asociados al uso de medios de protección frente a la radiación del láser.

\subsection{Comparación de fuentes láser en el soldeo de las series 5000 y 6000}

La soldabilidad depende de cada aleación, pero, en general, las aleaciones de la serie 6000 presentan mejores resultados que la 5000. De estas últimas, sólo se consiguen resultados aceptables en las de gran contenido de magnesio (p. ej., la AA5182), cuya evaporación durante la soldadura ayuda a mantener el keyhole abierto.

\subsubsection{YAG multi- $k W$ continuo frente a pulsado}

Las soldaduras realizadas con láseres continuos de Nd:YAG presentan buen aspecto del cordón y están libres de porosidad, cavidades o formación de grietas en caliente. Con láseres pulsados, el aspecto del cordón no es tan bueno (mayor pérdida de material y probabilidad de aparición de cavidades y porosidad), pero se consigue mayor penetración. Se han presentado en (2) ensayos de penetración y uniones a tope y a solape sobre AA6061 con YAG de $2 \mathrm{~kW}$ continuo, así como uniones en esquina sobre AA6061 y AA6082, realizados con $2 \mathrm{~kW}$ continuo y con láser de $3 \mathrm{~kW}$ pulsado. Otros estudios (3) con láseres pulsados muestran soldaduras libres de agrietamiento sobre $\mathrm{AlMg}_{0,4} \mathrm{Si}_{1,2}$, cuando el material se precalienta a $250{ }^{\circ} \mathrm{C}$.

$\mathrm{El}$ agrietamiento en caliente se produce durante la solidificación. El procesado con pulsos induce discontinuidades en el baño del fundido (tanto la soldadura láser como el aluminio favorecen una solidificación muy rápida) y facilita también la aparición de cavidades. La reducción en la aparición de cracks con ambos tipos de láseres mejora con una mayor velocidad de proceso. Aun con fuente continua son necesarios un cuidadoso alineamiento de los bordes a unir y un buen control de los parámetros.

Se ha ensayado la combinación de pulsos procedentes de fuentes diferentes, intercalando los pulsos para conseguir alta frecuencia de repetición; también se han superpuesto los pulsos para obtener finalmente pulsos de alta potencia. Así, el LZH 
(Hannover) (3) ha utilizado 3 láseres de $500 \mathrm{~W}$; el ILT (Aquigrán) puede combinar 3 láseres de $1 \mathrm{~kW}$ (2). La calidad continúa siendo inferior que con el proceso continuo. De igual forma, ocurre con la superposición de un haz pulsado sobre uno continuo (pulser-sustainer).

\subsubsection{YAG frente a $\mathrm{CO}$, fuentes continuas}

Para obtener la misma penetración en piezas de aluminio con estas fuentes, con una misma velocidad de proceso, la potencia necesaria es menor con láser de Nd:YAG (p. ej., 1/3 menor en AA6082). La estabilidad del proceso es mucho mayor en el caso del YAG: si se mide la estabilidad como distancia media entre cráteres en una pieza, las soldaduras con láser de Nd:YAG tienen 5 veces mayor estabilidad. Estas diferencias son función de las longitudes de onda.

La utilización de hilo de aporte mejora sensiblemente la estabilidad del proceso, pero no cambia la relación entre las estabilidades de los dos tipos de fuentes láser. Hay que hacer notar que en otros materiales (aceros) esa gran diferencia no existe. Con velocidades pequeñas el $\mathrm{CO}_{2}$ es más adecuado (4).

\section{COMBINACIÓN DE HACES DE ALTA POTENCIA}

En este capítulo se describen los resultados obtenidos en el IFSW con la combinación de 2 haces de potencia transmitidos por fibra. Los resultados de soldadura con dos focos extienden el rango de aplicación del láser de Nd:YAG y su flexibilidad en la soldadura de materiales como el aluminio. En principio, se persigue sólo aplicar más potencia a la pieza y conseguir mayor penetración y velocidad de proceso, pero los resultados son mejores que los obtenidos con un sólo haz de potencia igual a la suma de la de los dos haces: se incrementan la estabilidad del proceso y la calidad de la soldadura.

Cada haz es transmitido por una fibra; estas fibras se colocan paralelas y muy juntas, y a la salida se focalizan los haces 1:1 con óptica convencional, formando 2 focos sobre la pieza (Fig. 1). En (5), se describe otra estrategia: los haces se introducen en la misma fibra; a la salida, el haz se focaliza de forma convencional. La fibra sufre pronto daños y debe tener un diámetro grande $(1 \mathrm{~mm})$, con lo que los resultados son peores.

Con láseres de $\mathrm{CO}_{2}$ había sido probada (IFSW) con éxito la focalización independiente de los haces (utilizando óptica especial para cada haz), tanto sobre 1 mismo punto en la pieza, como en 2 puntos muy cercanos. Los haces están cruzados y se reduce la profundidad de campo, pero la buena calidad de
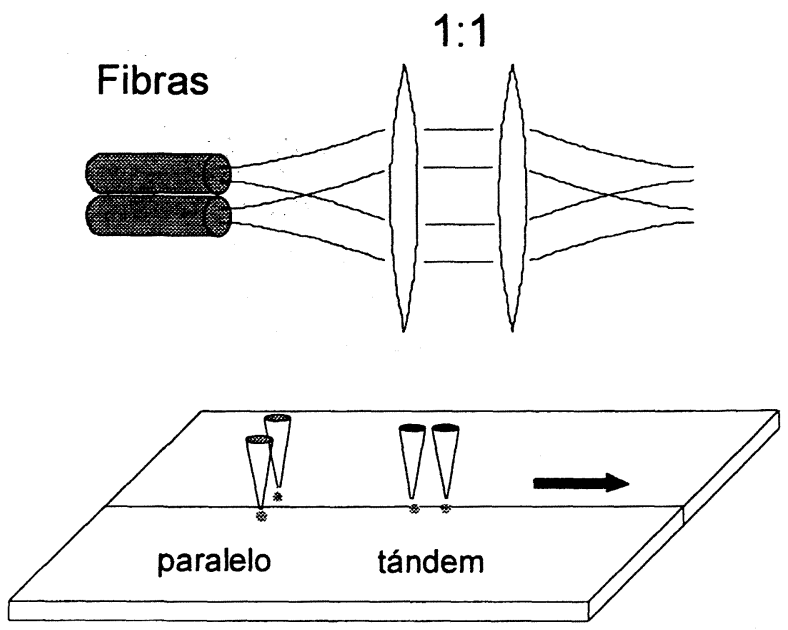

FIG. 1.- Uso de combinación de haces guiados por fibra.

\section{FIG. 1.-Dual beam arrangement.}

haz del $\mathrm{CO}_{2}$ permite su uso. La división de un haz, para su posterior combinado con dos focos, ha mostrado algunas ventajas respecto al haz sencillo. El guiado por fibra en Nd:YAG, simplifica el concepto.

Los resultados sobre AA5182 y AA6110 de 5 $\mathrm{mm}$ de espesor, con focal $100 \mathrm{~mm}$, son:

a. Distancia entre los focos. Ligeramente superpuestos, sin formación de 2 capilares.

b. Diámetro del núcleo de las fibras (también diámetro de cada punto focal). El uso de fibras de 400 ó $600 \mathrm{~mm}$ no afecta sensiblemente la penetración, pero con $400 \mathrm{~mm}$ es mayor la eficiencia del proceso (sección transversal del fundido). Con fibras de 200 $\mathrm{mm}$, la penetración se incrementa de forma sensible; sus resultados mejoran a altas velocidades.

c. Tipo de configuración (paralelo o tándem). No hay grandes diferencias en la penetración, cuando el resto de parámetros es óptimo. El cordón es más ancho en la configuración en paralelo, lo que tiene interés en soldeo a solape.

El uso de dos focos separados, con un solo keyhole, influye sobre la forma de éste y lo mantiene abierto. Da mayor estabilidad al keyhole y a la dinámica del fundido.

El gas de protección proporciona una atmósfera inerte alrededor del fundido y es fundamental en soldadura. Hay que destacar que con la combinación de haces con fibras de $200 \mathrm{~mm}$ no aparecen diferencias claras en el uso de un gas u otro. Con fibras mayores, el helio presenta los mejores resultados. 
El hilo de aporte tiene también un papel estabilizador del fundido y añade al cordón los elementos que el material base pierde en el soldeo. Se utilizó $\mathrm{AlSi}_{\mathrm{x}} \mathrm{Mg}_{\mathrm{y}} \mathrm{Mn}$, probando distintas composiciones de silicio y magnesio. El silicio contrarresta la pérdida de propiedades mecánicas debidas a la evaporación de magnesio, pero su adición limita la elasticidad. Con el $\mathrm{AlSi}_{7} \mathrm{Mg}_{0,6} \mathrm{Mn}$ se obtuvieron las mejores propiedades mecánicas de la unión en la serie 6000 . Para AA5182, el hilo más adecuado fue $\mathrm{AlSi}_{5} \mathrm{Mg}_{0,4} \mathrm{Mn} .10$

La resistencia a la tracción y el límite elástico de las aleaciones de la serie 6000 disminuyen a un 50$60 \%$ de las del material base, en tanto que en la serie 5000 se mantienen en un $80 \%$.

Como conclusiones, la utilización de 2 fuentes de Nd:YAG, como una sola, proporciona un aumento de la estabilidad (calidad) en la soldadura de aluminio. Además, permite utilizar potencias no disponibles con fuentes láser individuales.

\section{EMISIONES EN SOLDADURA LÁSER DE ND:YAG DE ALTA POTENCIA}

Se presentan los resultados de la emisión de partículas durante el proceso. Se ha caracterizado la materia particulada emitida al aire durante el procesado (soldadura a tope y ensayos de penetración) de 3 aleaciones de Al: AA5182, AA6060 y AA6110 con láser continuo de Nd:YAG de $2 \mathrm{~kW}$. Se determinaron:

- concentración de aerosoles en conducto de aspiración local

- composición química de elementos metálicos

- distribución de tamaño de las partículas

El haz láser (Fig. 2) es guiado por fibra óptica hasta el cabezal de salida, que se desplaza sobre las piezas a procesar movido por un robot antropomórfico de 5 ejes. Las probetas de la serie 6000, de $2 \mathrm{~mm}$ de espesor, se procesaron con velocidad de $3 \mathrm{~m} / \mathrm{min}$. La aleación AA5182, de 2,3 mm de espesor, se soldó a $2,5 \mathrm{~m} / \mathrm{min}$. Se describen aquí los experimentos en que se utilizó hilo de aporte $\mathrm{AlSi}_{7} \mathrm{Mg}_{0,6} \mathrm{Mn}$ de $1 \mathrm{~mm}$ de diámetro con velocidad $3 \mathrm{~m} / \mathrm{min}$. El gas de asistencia $(\mathrm{He})$ se aporta fuera del eje del haz. El aire comprimido, aplicado transversalmente, protege a la óptica de las proyecciones de aluminio.

La concentración de aerosoles se midió dentro del conducto de aspiración local; éste se mueve con el cabezal láser y está colocado de forma que la extracción sea máxima. En la tabla I, se muestra la concentración de partículas en conducto (útiles en el diseño de sistemas de aspiración) y las correspondientes tasas de emisión recogidas en los ensa-

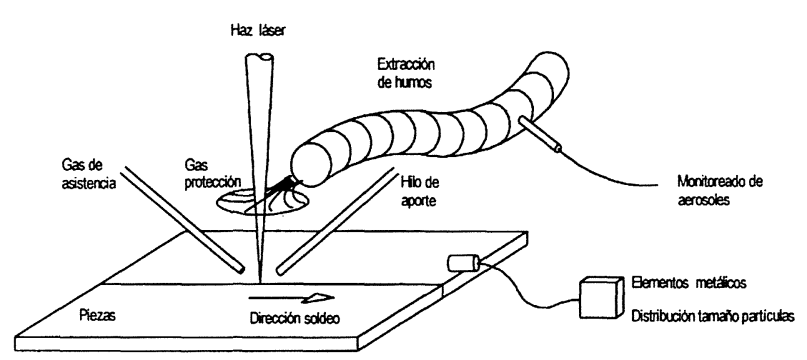

FIG. 2.- Monitorización de las emisiones de soldadura.

FIG. 2.-Sampling the emissions from the welding process.

Tabla I.- Concentración de aerosoles y tasas de emisión por metro de soldadura y por minuto.

TABLE I.-Aerosol concentration and emission rates.

\begin{tabular}{|c|c|c|c|}
\hline & $\mathrm{mg} / \mathrm{m}^{3}$ & $\mathrm{mg} / \mathrm{m}$ & $\mathrm{mg} / \mathrm{min}$ \\
\hline AA518 & 22,59 & 8,58 & 21,46 \\
AA606 & 14,13 & 5,37 & 16,11 \\
AA611 & 16,29 & 6,19 & 18,58 \\
\hline
\end{tabular}

yos de soldadura a tope. En las tres aleaciones, se obtiene un $23 \%$ más de emisiones en las soldaduras a tope que en los correspondientes cordones realizados directamente sobre la pieza. Esto se debe a la mayor superficie libre de la unión a tope.

La medición de aerosoles se realizó también en zonas alejadas unos $3 \mathrm{~m}$ del lugar de proceso, a una altura similar a la de las entradas respiratorias de un técnico. Los valores de concentración están en torno al $0,75 \%$ de las medidas cerca del proceso.

Los análisis de composición química de elementos metálicos muestran (Tabla II) valores más altos para el aluminio, el magnesio y el silicio, de acuerdo con la composición de las aleaciones. La materia particulada tiene mayor proporción de magnesio (en la serie 6000, también de silicio) que el material base, como corresponde a los puntos de ebullición de cada elemento. Las tres primeras filas son los valores medios obtenidos en la zona de proceso; las dos últimas filas corresponden a las proporciones del muestreo en la zona alejada del proceso.

TABLA II.- Composición de elementos metálicos (\%)

TABLE II.- Metallic element composition (\%)

\begin{tabular}{|c|c|c|c|c|c|c|}
\hline & $\mathrm{Cr}$ & $\mathrm{Fe}$ & $\mathrm{Mn}$ & $\mathrm{Mg}$ & $\mathrm{Al}$ & $\mathrm{Si}$ \\
\hline AA518 & 0,0 & 1,52 & 0,0 & 21,3 & 49,6 & 27,4 \\
AA606 & 0,52 & 4,63 & 0,0 & 15,4 & 38,6 & 40,5 \\
AA611 & 0,51 & 2,92 & 0,25 & 20,4 & 55,5 & 20,3 \\
AA518 & 0,20 & 1,01 & 0,20 & 30,1 & 60,8 & 7,54 \\
AA6xx & 0,82 & 1,63 & 0,0 & 14,7 & 50,0 & 32,7 \\
\hline
\end{tabular}


La mayor parte de las partículas son $<1 \mu \mathrm{m}$ en todos los materiales y prácticamente todas son $<6$ $\mu \mathrm{m}$. La fracción respirable para partículas $<1 \mu \mathrm{m}$ es del $97 \%$, y el $50 \%$ para menores de $5 \mu$ m (6). La emisión de partículas de mayor tamaño en la aleación AA5182 y sus ratios de emisión algo superiores, son debidas a su peor soldabilidad.

\section{Agradecimiento}

Los autores agradecen al INSHT y al Laboratorio de Higiene Industrial de FREMAP su ayuda en los análisis y sus facilidades en el uso de material de muestreo. Este trabajo se realizó nominalmente desde CETEMA, dentro del proyecto BE-7997 (Welding of advanced materials with high-power $C W$ and Pulser-sustainer YAG lasers). Asimismo agradecen al Institute für Strahlwerkzeuge (IFSW) su colaboración y la autorización para utilizar sus instalaciones.

\section{REFERENCIAS}

(1) ANDERSON, S.G. Laser Focus World. Ene. 1998: 78-98.

(2) Garcia, L.M., Sanchez, L. y Garcia, J.A. Sold. Tecnol. Unión, 41, Oct.1996: 28-36.

(3) Beske, E.U., Schumacher, J. y Kreutzburg, K. Joining of sheet metals with poor weldability using $\mathrm{kW} \mathrm{Nd:YAG}$ lasers. ICALEO 1993: 682-691.

(4) Päthe, D., Roatzsch, R., Barthel, W. y Weick, J.M. Influence of beam quality when welding with Nd:YAGand $\mathrm{CO}_{2}$ - lasers. Proc. Europto 1997. SPIE Vol. 3.097.

(5) Bostanjoglo, G., Beck, T. y Richter, K. 6-kW Nd:YAGlaser-system for welding applications. Proc. Europto 1997. SPIE Vol.3.097.

(6) Size fraction definitions for measurement of airborne particles EN-481:1993. 